\title{
Ground Disasters Visual Representation Based on Multi-Field Geospatial Infographics Spectra in Mining Area
}

\author{
Zhang Jin ${ }^{\mathrm{a}, *}$ \\ ${ }^{a}$ Taiyuan University of Technology, Taiyuan, Shanxi, P. R. China, 030024, zjgps@163.com \\ * Corresponding author
}

Keywords: Multi-Field information, Geospatial infographics spectra, Ground Disasters, Visualization Representation, Coal Mine

\begin{abstract}
:
Geospatial infographics spectra is a new cross research direction of cartographic theory. It combines the infographic representing geospatial information with the spectra recording spatial-temporal evolution to form geo-infographics spectra, which reflects the characteristics and dynamic changes of the object's morphological structure, mechanism, composition, and reveals the temporal and spatial variation law of regional geo-phenomena. Infographics can be a map in the form of spatial information. It also includes other graphical forms such as images, charts, curves and so on. Spectra is a system established by object characteristics or by time series. Geospatial infographics spectra has the dual characteristics of graphics and genealogy. It is a spatial-temporal composite analysis method that visually represents the spatial morphological structure and spatial-temporal changes of the earth system and its elements and phenomena through graphical thinking and abstract generalization of multi-field geo-digital information.
\end{abstract}

From geospatial morphological characteristics, spectrum characteristics, texture characteristics, topographic characteristics, deformation characteristics, structural characteristics, geological environment characteristics, mining characteristics, space-time characteristics, disaster chain characteristics and other dimensions to understand and perspective in depth for geo-disasters in mining areas, to achieve the location-morphology-attribute-semanticsstructure-process-relationship of ground disaster based on geo-infographic spectra coupling representation, to construct the geo-disaster environment model expression based on surface functional zoning and geographical grids for mining areas. See figure 1 .

The spatial morphologies, structure, status, movement, subsidence, stress-strain, etc in the characteristic area of ground hazards is generalized as the basic field (the shape, structure, image information, topography of the hazard body). All the fields that affect the basic field are called effecting field (deformation field, geological structure field, geophysical field, stress field, surface cover field, rainfall field, temperature field, seepage field, soil chemical field, electromagnetic field, etc.). The third is coupled fields, that is scalar or vector fields formed by the coupling of the basic and effecting field (coupling of deformation field and topographic field, deformation field and seepage field, etc.). Based on the 3D mine field model and multi-field information such as basic field, effecting field and coupling field, first we can analyse the time series development stages of stable, oscillating, multi-step, catastrophic, trend-type disasters and then quantitatively segment, partition the types of deformation curves such as V-type, B-type, D-type, R-type pendulum-type and finally set index, discovery pattern and generate geospatial infographics spectra. See figure 2 and figure 3 .

A multi-dimensional features-based ground disaster data perception model integrating time, space, semantics and relationships is constructed to realize multi-field information and multi-granularity parsing and location-based information aggregation in disaster feature area. The automatic location information discovery and matching, location semantics association and spatial behaviour inference are accomplished. The spatial behaviour inference under different location semantics is supported to reveal coal mining. It provides precise information support for the impact of the surface and the mechanism of dynamic change. See figure 3.

\begin{tabular}{|c|c|c|c|c|c|c|c|c|}
\hline \multirow{3}{*}{$\begin{array}{l}\text { The Features } \\
\text { Of Geo-Disasters } \\
\text { Association }\end{array}$} & \multicolumn{2}{|c|}{$\begin{array}{l}\text { Location and } \\
\text { Relationship }\end{array}$} & \multicolumn{2}{|c|}{$\begin{array}{l}\text { Spatio-Temporal } \\
\text { Process }\end{array}$} & \begin{tabular}{|c|} 
Spatial \\
Distributions
\end{tabular} & $\begin{array}{c}\text { Spatial } \\
\text { Structure }\end{array}$ & \multicolumn{2}{|c|}{\begin{tabular}{|c|} 
Stratigraphic structure \\
and lithology
\end{tabular}} \\
\hline & Type & \multicolumn{2}{|c|}{ Pattern and Shape } & & vel of Details & \multicolumn{3}{|c|}{ Composition and Decomposition } \\
\hline & \multicolumn{2}{|c|}{ Land Coverage } & \multicolumn{2}{|c|}{ Geomorphometry } & Disaster Chain & \multicolumn{2}{|c|}{ Deformation } & Coal mining \\
\hline
\end{tabular}

Figure 1 The features of geo-disasters association 


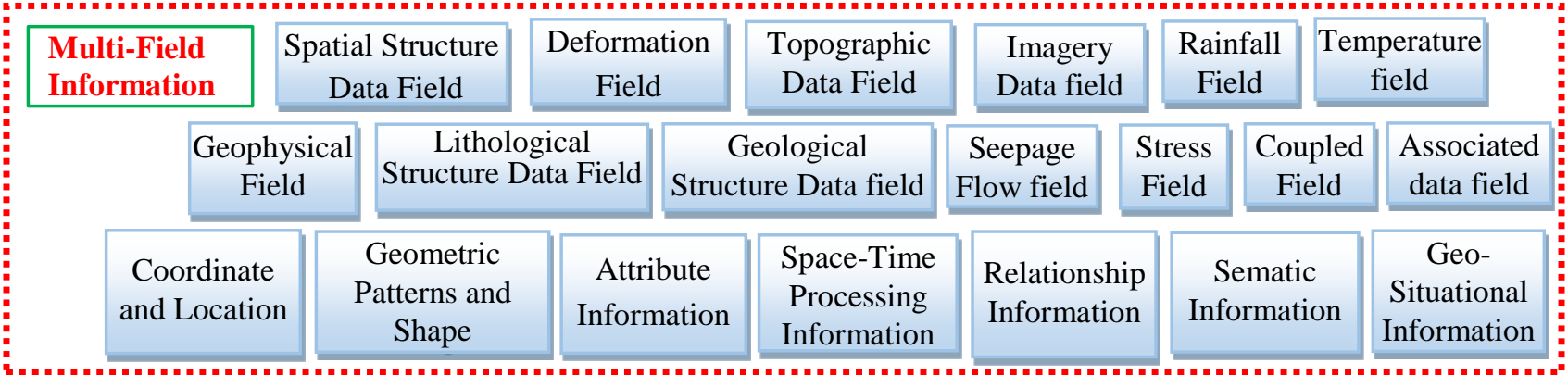

Figure 2 Multi-Field Geospatial Information
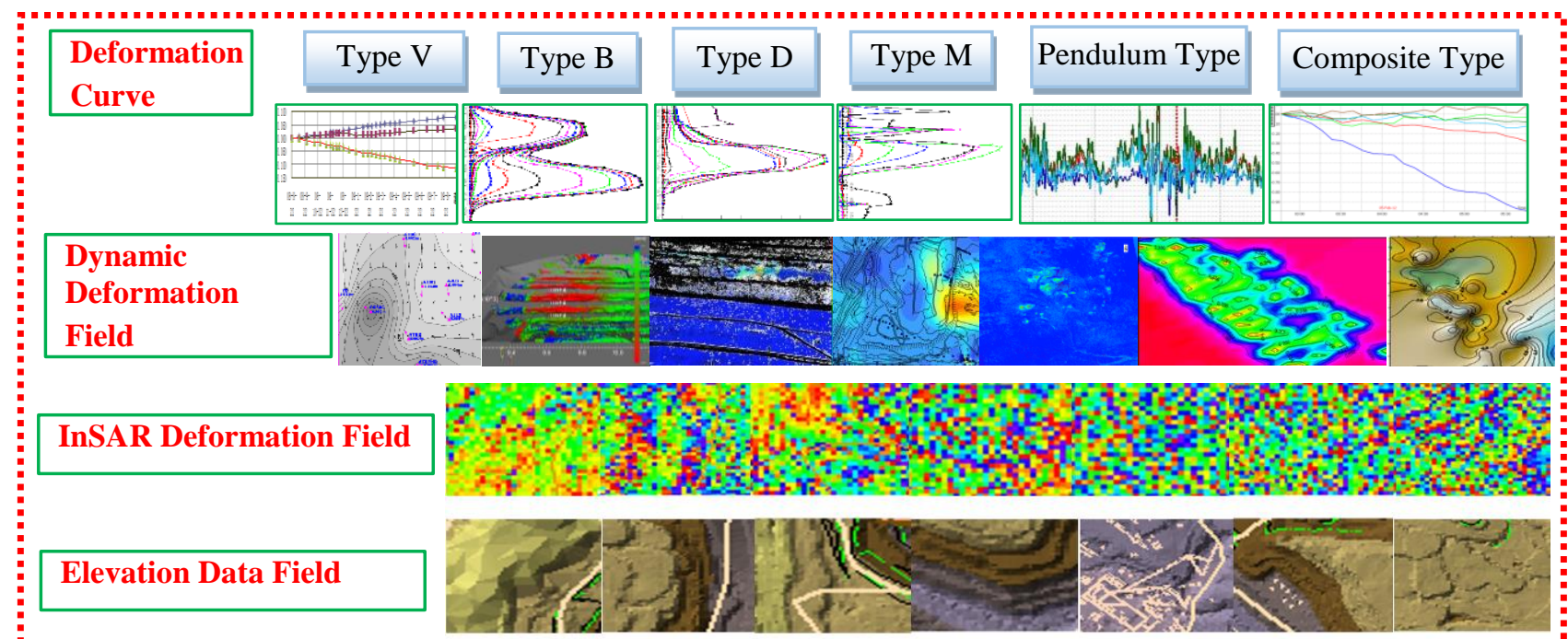

\begin{tabular}{|l|}
\hline Imagery Data Field \\
\hline Geographical Scene \\
Data Field \\
\hline Terrestrial Scanner \\
Data Field \\
\hline
\end{tabular}

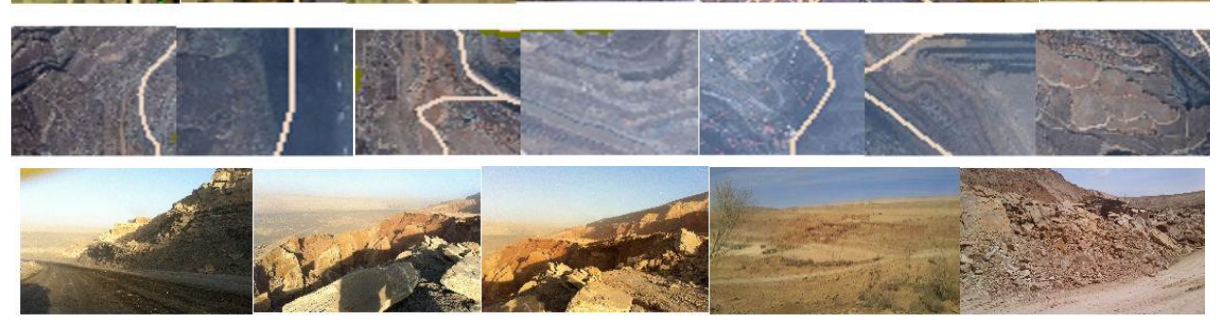

Data Field

\section{D Visualization \\ Data Field}
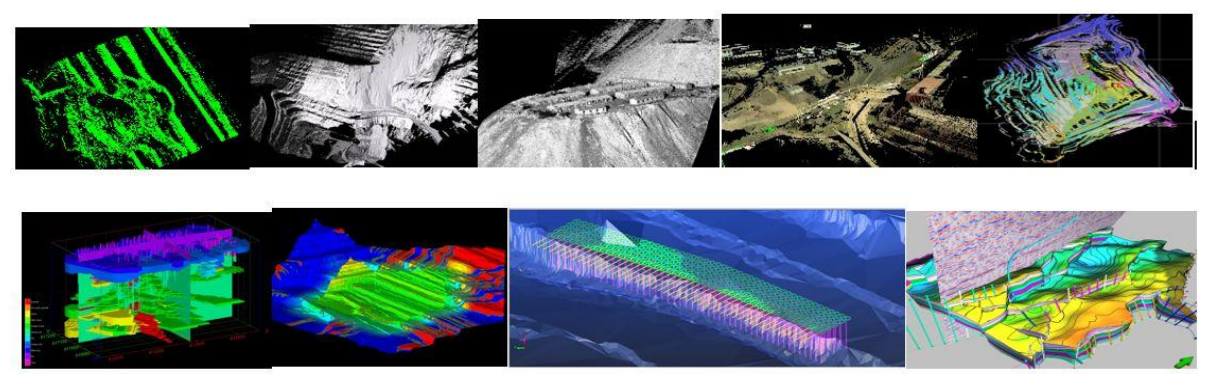

\begin{tabular}{l} 
Spatial Temporal Process \\
infographic spectrum \\
Data Field \\
\hline \begin{tabular}{l} 
Geo-infographic \\
spectrum \\
Location Association \\
\hline
\end{tabular}
\end{tabular}

Figure 3 The Multi-field geo-infographic spectra of ground disasters in large open-pit mining area

\section{Acknowledgment :}

This work was supported by the National Natural Science Foundation of China (Grant No. 41771443) and the National Key Research and Development Program (Grant No. 2016YFB0502601、2018YFB0505402). 